\title{
Interferon- $\alpha$ enhances the susceptibility of renal cell carcinoma to rapamycin by suppressing mTOR activity
}

\author{
XIAO HAN, DONGHAO SHANG, TIANDONG HAN, XIUHONG XU and YE TIAN \\ Department of Urology, Beijing Friendship Hospital, Capital Medical University, Beijing 100050, P.R. China
}

Received October 29, 2013; Accepted March 19, 2014

DOI: $10.3892 /$ etm.2014.1691

\begin{abstract}
The aim of the present study was to investigate the antiproliferative effects of interferon (IFN)- $\alpha$ and rapamycin (RPM) on renal cell carcinoma (RCC) cells and examine the synergistic growth suppression conferred by IFN- $\alpha$ and RPM. The effects of IFN- $\alpha$ and/or RPM on RCC cells were determined using a WST-1 assay and the synergy of IFN- $\alpha$ and RPM against three RCC cell lines was analyzed with isobolographic analysis. The expression of mammalian target of rapamycin (mTOR) was downregulated by RNAi, and the expression and phosphorylation of proteins in the mTOR pathway following treatment with IFN- $\alpha$ and/or RPM was examined by western blot analysis. The observations indicated that IFN- $\alpha$ significantly increased the susceptibility of RCC cells to RPM and the synergistic effect of IFN- $\alpha$ and RPM against RCC cells was confirmed in all three RCC cell lines. The mTOR pathway was shown to be associated with the synergistic effect of IFN- $\alpha$ and RPM against RCC. IFN- $\alpha$ and RPM alone decreased the phosphorylation of mTOR, p70 S6 kinase, S6 and 4E binding protein 1 , and IFN- $\alpha$ significantly enhanced the RPM-induced suppression of the mTOR pathway. However, in RCC cells with low mTOR activity, the synergy of IFN- $\alpha$ and RPM was eliminated. Therefore, the results of the present study indicate that the mTOR pathway plays an important role in the synergistic effect of IFN- $\alpha$ and RPM against RCC cells. Thus, mTOR may serve as an effective therapeutic target in the treatment of advanced RCC.
\end{abstract}

\section{Introduction}

Renal cell carcinoma (RCC) is the most common type of kidney cancer in adults. Following the occurrence of metastasis, survival rates are very poor and the 5-year survival rate is $20 \%$ (1). RCC is resistant to chemotherapy (2). At

Correspondence to: Professor Ye Tian, Department of Urology, Beijing Friendship Hospital, Capital Medical University, 95 Yongan Road, Beijing 100050, P.R. China

E-mail: yetian79@163.com

Key words: renal cell carcinoma, interferon- $\alpha$, rapamycin, mammalian target of rapamycin present, treatment regimens using interferon (IFN)- $\alpha$ have been applied in clinical practice to treat RCC, achieving therapeutic response rates between 4 and 33\% (3). A previous study revealed that IFN- $\alpha$ mediates anticancer effects indirectly by modulating immunomodulatory mechanisms or directly through antiproliferative effects and inducing the differentiation of cancer cells (4).

IFN- $\alpha$ exerts these effects by binding to cell surface receptors and activating the Janus kinase (Jak) protein family. Activated Jak1 and tyrosine kinase 2 phosphorylate signal transducers and activators of transcription (STATs). Subsequently, phospho-STATs translocate to the nucleus and interact with specific regulatory elements to induce target gene transcription (5). RCC treatment has developed significantly, as vascular endothelial growth factor (VEGF) receptor tyrosine kinase inhibitors and drugs that inhibit mammalian target of rapamycin (mTOR) signaling have become the mainstay for the management of advanced RCC. These treatments have improved progression-free survival and/or overall survival outcomes (6). The mTOR pathway has been reported to be central to cancer progression and rapamycin (RPM) has been shown to suppress carcinogenesis by decreasing mTOR activity (7). RPM may function by stimulating the degradation of cyclin D1, which inhibits the G1 to S-phase transition in the cell cycle (8). RPM also downregulates phospho-p70 S6 kinase $(\mathrm{K})$, which is considered to be an indicator of the activated mTOR pathway (9). The primary substrate of $\mathrm{p} 70$ S6K, S6 ribosomal protein, has also been shown to have an important role in determining cell size. Phosphorylation of the eukaryotic translation initiation factor, 4E binding protein 1 (4E-BP1), by mTOR results in the activation of cap-dependent translation of nuclear mRNAs by releasing the inhibition of the eukaryotic translation initiation factor 4E (10). RPM has been shown to suppress the growth of small cell lung cancer and pancreatic cancer cells $(11,12)$. In addition, mTOR inhibitors have shown promising efficacy in early-stage trials in patients with advanced RCC (13). A previous study indicated that RPM may be of value to patients with RCC and that the antitumor efficacy of RPM is achieved by cell-cycle arrest and targeted reduction of VEGF-A and transforming growth factor- $\beta 1$ (14). An additional study revealed the synergistic effects of RPM and chemotherapeutic agents against tumor cells; RPM was reported to increase the cytotoxicity of cisplatin by sensitizing human promyelocytic leukemia and ovarian cancer cells to the drug, thereby inducing apoptosis (15). However, receptor 
tyrosine kinase inhibitors only demonstrate additive effects in combination with RPM in the treatment of prostate cancer (16). A previous study indicated that IFN- $\alpha$ suppresses the phosphoinositide 3 kinase and mTOR signaling pathways (17). Furthermore, combining RPM with other upstream mTOR inhibitors has been shown to induce greater growth suppression in RCC compared with that achieved by administering the drugs alone (18). However, whether IFN- $\alpha$ and RPM have a synergistic effect against RCC remains unknown.

High frequency mutations or the loss of the two copies of the Von Hippel-Lindau (VHL) tumor suppressor gene have been observed in RCC (19). VHL protein is the substrate recognition component of the $\mathrm{E} 3$ ligase that ubiquitinates hypoxia-inducible transcription factors (HIFs), including HIF- $1 \alpha$ and $-2 \alpha$. VHL plays a pivotal role in the downregulation of VEGF expression (20). Previous studies have indicated that mTOR stimulates HIF expression and RPM exhibits antiangiogenic activity that is associated with a reduction in the production of HIF/VEGF $(21,22)$. However, the effect of VHL activity on the antiproliferative ability of IFN- $\alpha$ and RPM in RCC remains unknown.

\section{Materials and methods}

Cell lines and agents. Three RCC cell lines, ACHN, NC65 and A498 (ATCC, Rockefeller, MD, USA), were cultured in complete medium consisting of RPMI-1640 (Gibco, Gaithersburg, MD, USA) supplemented with $25 \mathrm{mM}$ hydroxyethyl piperazineethanesulfonic acid, $2 \mathrm{mM}$ L-glutamine, $1 \%$ nonessential amino acids, $100 \mathrm{U} / \mathrm{ml}$ penicillin, $100 \mu \mathrm{g} / \mathrm{ml}$ streptomycin and $10 \%$ heat-inactivated fetal bovine serum. Cell lines were maintained as monolayers on $10-\mathrm{cm}$ plastic dishes and incubated in a humidified atmosphere containing $5 \% \mathrm{CO}_{2}$ at $37^{\circ} \mathrm{C}$. Intron A (recombinant IFN- $\alpha 2 b$ ) was purchased from Merck \& Co, Inc. (Whitehouse Station, NJ, USA) and RPM was purchased from Sigma-Aldrich (St. Louis, MO, USA).

WST-1 assays. Effects of IFN- $\alpha$ and/or RPM on the RCC cells were determined using a WST-1 assay. Exponentially growing cells were harvested and seeded at 2,000 cells/well in a 96-well microtiter plate. After $4 \mathrm{~h}$ of incubation, Intron A (10, 50, 100, $200,400$ or $800 \mathrm{IU} / \mathrm{ml})$, RPM $(1,5,10,15$ or $20 \mu \mathrm{M})$, a combination of Intron A (50 or $100 \mathrm{IU} / \mathrm{ml})$ and RPM (1, 5, 10, 15 or $20 \mu \mathrm{M}$ ), or penicillin/streptomycin medium (untreated control) were added. The cells were then continuously incubated for 72 h. WST-1 (Roche Diagnostics, Penzberg, Germany) at a volume of $10 \mu \mathrm{l}$ was added to each well and the cells were incubated for an additional $2 \mathrm{~h}$. Absorbance was measured with a microculture plate reader (Immunoreader; Japan Intermed Co., Ltd., Tokyo, Japan) at $450 \mathrm{~nm}$. The percentage of cell cytotoxicity was calculated using the following formula: $\%$ Cytotoxicity = [1 - (absorbance of experimental - absorbance of blank)/(absorbance of untreated control - absorbance of blank)] x 100.

siRNA transfection. A498 cells, which lack the wild-type VHL gene, were stably transfected using Lipofectamine 2000 (Invitrogen Life Technologies, Carlsbad, CA, USA) with an expression vector containing the full-length cDNA for VHL or with a blank vector without the VHL insert. Single colo- nies were selected with G418 and confirmed by cell staining, western blot analysis and cDNA sequencing. ACHN and A498 cells were seeded in complete medium without antibiotics and were allowed to grow until 30-50\% confluence was reached. The cells were then transfected with siRNA oligonucleotides or scrambled siRNA control using Lipofectamine 2000. Following incubation for $72 \mathrm{~h}$, gene expression was confirmed by western blot analysis. SignalSilence mTOR siRNA I was purchased from Cell Signaling Technology, Inc. (Beverly, MA, USA). All RNAi target sequences and oligonucleotide sets used in the study are shown in Table I.

Reverse transcription polymerase chain reaction (RT-PCR). Total RNA was isolated using an RNeasy mini kit (Qiagen, Frankfurt, Germany). A first-strand cDNA synthesis kit (GE Healthcare, Little Chalfont, UK) was used for reverse transcription. The PCR conditions were selected according to the manufacturer's instructions and the expected sizes of the PCR products were confirmed by agarose gel electrophoresis. The PCR products were quantified with a GeneAmp 5700 Sequence Detection system (Applied Biosystems, Inc., Foster City, CA, USA). All primer sets used in this study are shown in Table I.

Western blot analysis. The procedures were performed as previously described (23). Protein was extracted and the concentration was measured using a Bradford dye-binding protein assay (Bio-Rad Laboratories, Inc., Richmond, CA, USA). Subsequently, SDS polyacrylamide gel electrophoresis was performed. Anti- $\beta$-actin monoclonal antibodies (Abcam, Cambridge, UK) were used as an internal control. Other antibodies used in the study were all purchased from Cell Signaling Technology, Inc.. These included mTOR (7C10)/phospho-mTOR (Ser2481), p70 S6K (49D7)/phospho-p70 S6K (Thr421/Ser424), S6 ribosomal protein (5G10)/phospho-S6 ribosomal protein (Ser240/244) (D68F8) XP and 4E-BP1 (53H11)/phospho-4E-BP1 (Thr70) rabbit monoclonal antibodies. Immune complexes were detected using an enhanced chemiluminescence system (GE Healthcare) combined with image analysis. The image analysis software used was ImageJ (NIH, Bethesda, MD, USA).

Statistical analysis. All determinations were performed in triplicate and the results are expressed as the mean \pm SD. Statistical significance was determined using the Student's t-test and $\mathrm{P}<0.05$ was considered to indicate a statistically significant difference. Synergy was evaluated by isobolographic analysis, as described by Berenbaum (24). The fractional inhibitory concentration of each agent was equal to the $\mathrm{IC}_{50}$ dosage of the agent in combination divided by the $\mathrm{IC}_{50}$ dosage of the agent when used alone. An additive, synergistic or antagonistic combination was indicated by whether the point lies on, below or above, respectively, the straight line joining the dosages of the two drugs that when administered alone produce the same effect as that of the combination, as based on the isobolographic analysis.

\section{Results}

Synergistic growth suppression by IFN- $\alpha$ and RPM. IFN- $\alpha$ administration caused dose-dependent cell growth inhibition in 
Table I. Primer and RNAi sequences.

A. Primer sequences

\begin{tabular}{llll}
\hline Gene & \multicolumn{1}{c}{ Forward primer, 5'-3' } & Reverse primer, 5'-3' & Length of PCR products, bp \\
\hline VHL & AGAAGGTGGTGGCATTTTTG & AGCAGATGCCAATGCCTTCT & 124 \\
HIF-1 $\alpha$ & GAAAGCGCAAGTCCTCAAAG & CATACGGTCTTTTGTCACTG & 126 \\
HIF-2 $\alpha$ & TTGATGTGGAAACGGATGAA & CTCATGGGGTTTTGGGTGAA & 110 \\
GAPDH & GAAGGTGAAGGTCGGAGTC & GAAGATGGTGATGGGATTTC & 226 \\
\hline
\end{tabular}

B. RNAi sequences

\begin{tabular}{lrrr}
\hline Gene & Sense oligonucleotide, 5'-3' & Antisense oligonucleotide, 5'-3' & Target gene sequence, 5'-3' \\
\hline VHL & CGAGCGCGCGCGAAGACUACG & UAGUCUUCGCGCGCGCUCGGU & ACCGAGCGCGCGCGA \\
& & & AGACTACG (98-120 bp) \\
Negative control & GUACCGCACGUCAUUCGUAUC & UACGAAUGACGUGCGGUACGU &
\end{tabular}

VHL, Von Hippel-Lindau; HIF, hypoxia-inducible transcription factor; PCR, polymerase chain reaction.

A

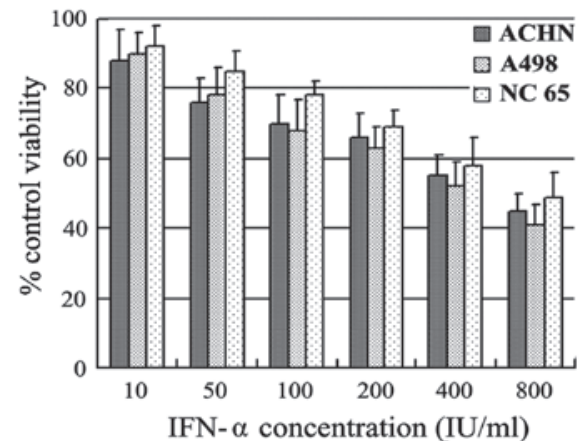

$\mathbf{C}$

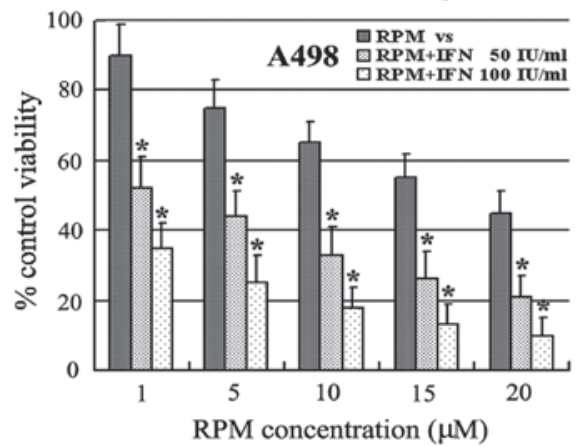

B

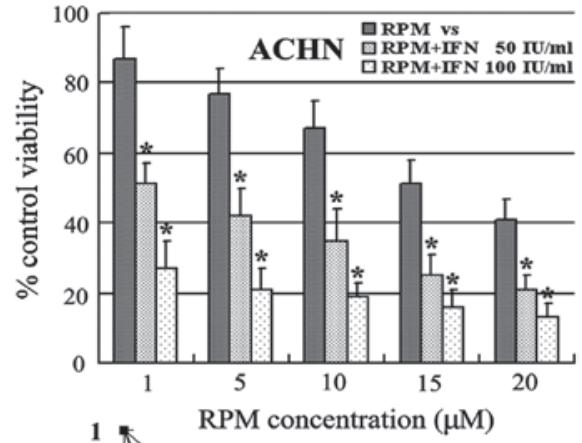

D

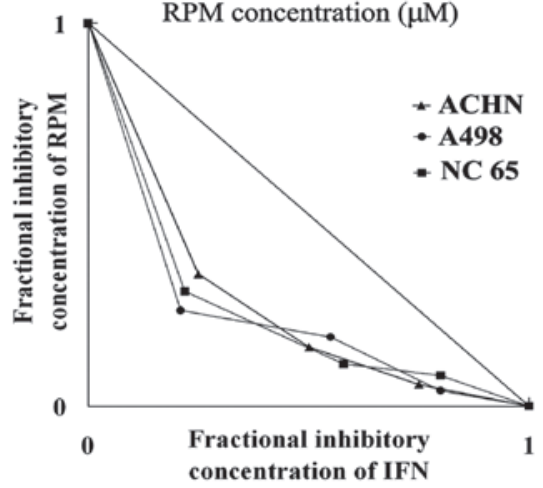

Figure 1. Synergy of IFN- $\alpha$ and RPM in RCC cell lines. Bar graphs showing the effect of (A) IFN- $\alpha$ on RCC cells, and the combination effect of IFN- $\alpha$ and RPM on (B) ACHN and (C) A498 cell proliferation. (D) Synergy of IFN- $\alpha$ and RPM in RCC cell lines was assessed by isobolographic analysis. All determinations were performed in triplicate and error bars represent SD. " $\mathrm{P}<0.01$ vs. RPM alone. IFN, interferon; RPM, rapamycin; RCC, renal cell carcinoma.

the ACHN, A498 and NC65 RCC cell lines (Fig. 1A). In addition, a combination of IFN- $\alpha$ and RPM caused dose-dependent cell growth inhibition in the RCC cell lines (Figs. 1B and C). IFN- $\alpha$, at low concentrations of 50 and $100 \mathrm{IU} / \mathrm{ml}$, significantly increased the susceptibility of the ACHN and A498 RCC cell lines to RPM (Fig. 1B and C). Combined treatment with IFN- $\alpha$ and RPM resulted in synergistic growth suppression in all the RCC cell lines examined in this study, as shown by isobolographic analysis (Fig. 1D).
Suppression of mTOR pathway components by IFN- $\alpha$ and/or $R P M$. To determine if the mTOR pathway is involved in the synergistic effect of IFN- $\alpha$ and RPM against RCC cells, phosphorylation of the mTOR pathway was evaluated following stimulation with IFN- $\alpha$ and/or RPM. In the ACHN and A498 cell lines, although $100 \mathrm{IU} / \mathrm{ml} \mathrm{IFN}-\alpha$ and/or $5 \mu \mathrm{M}$ RPM did not affect the total protein expression of mTOR, p70 S6K, S6 or 4E-BP1, it was observed that IFN- $\alpha$ and RPM, alone or in combination, decreased the phosphorylation of mTOR, 


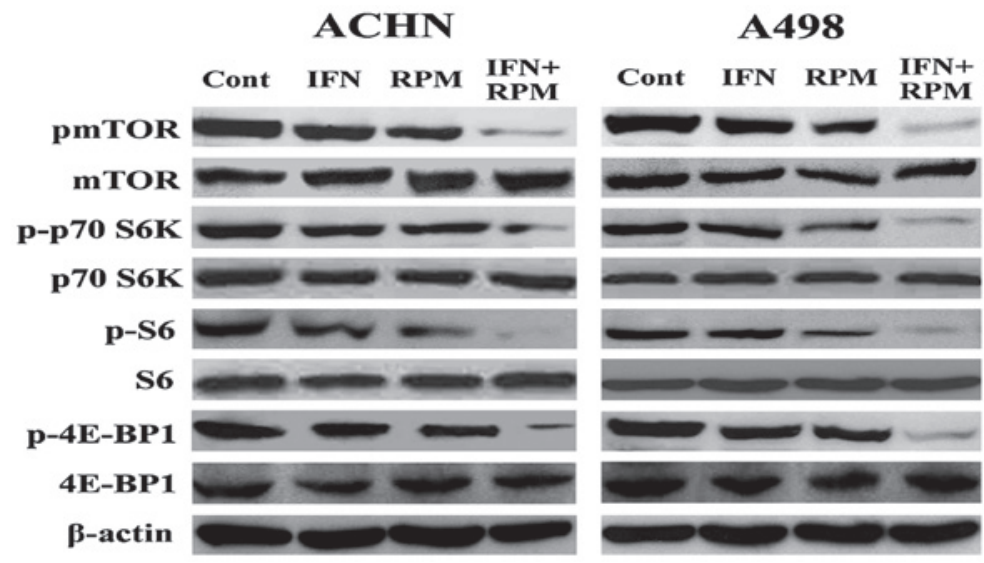

Figure 2. Suppression of the mTOR pathway by IFN- $\alpha$ and/or RPM. Western blot analysis demonstrates the effects of IFN- $\alpha$ and RPM, alone and in combination, on the ACHN and A498 RCC cell lines. mTOR, mammalian target of rapamycin; IFN, interferon; RPM, rapamycin; RCC, renal cell carcinoma.

A

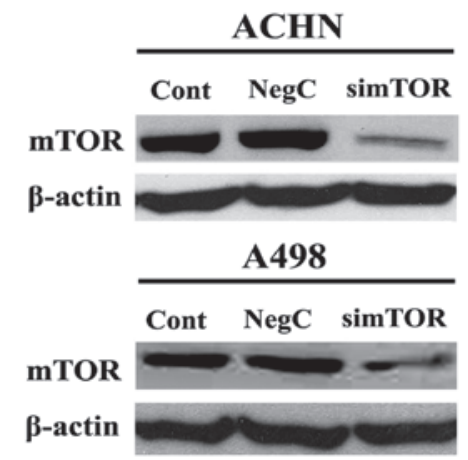

C

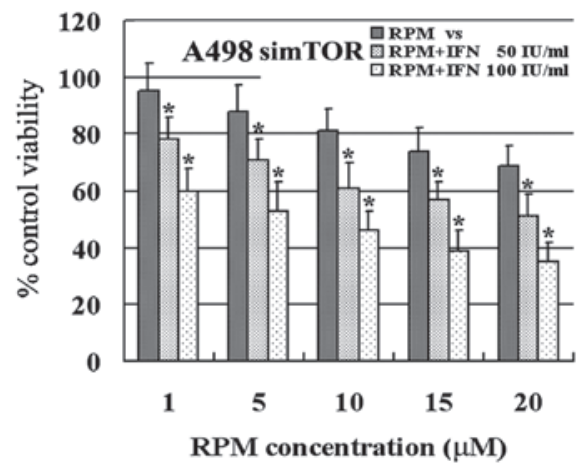

B

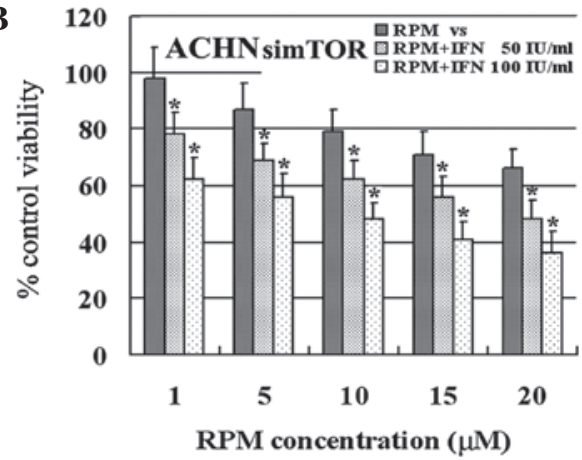

D

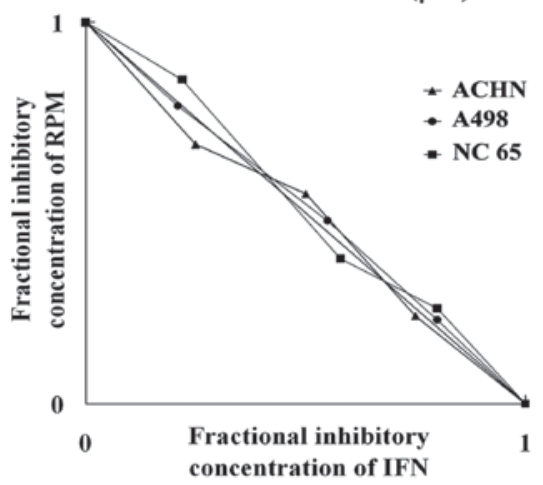

Figure 3. Effect of mTOR activity on the synergy of IFN- $\alpha$ and RPM. (A) Western blot analysis showing the expression of mTOR in two RCC cell lines transfected with RNAi. Bar graphs demonstrating the combination effect of IFN- $\alpha$ and RPM on m-TOR silenced (B) ACHN and (C) A498 RCC cells. (D) Effect of IFN- $\alpha$ and RPM on two m-TOR silenced RCC cell lines based on isobolographic analysis. All determinations were performed in triplicate and error bars represent SD. "P<0.01 vs. RPM alone. mTOR; mammalian target of rapamycin; IFN, interferon; RPM, rapamycin; RCC, renal cell carcinoma.

p70 S6K, S6 and 4E-BP1, as determined by western blot analysis (Fig. 2). In addition, IFN- $\alpha$ significantly enhanced the RPM-induced suppression of the mTOR pathway in these two cell lines. These results indicate that the mTOR pathway plays a key role in the synergistic effect of IFN- $\alpha$ and RPM against RCC cells.

Effect of mTOR activity on the synergy of IFN- $\alpha$ and RPM. The effect of mTOR activity on the synergy of IFN- $\alpha$ and RPM against RCC was investigated. The expression of mTOR was downregulated by RNAi and the results indicated that mTOR expression was suppressed effectively in ACHN and A498 cells (Fig. 3A). Regardless of mTOR expression, IFN- $\alpha$ enhanced the susceptibility of RCC to RPM in ACHN and A498 cells (Fig. 3B and C). However, the synergy of the two agents was eliminated in these cell lines, as an additive effect was indicated by isobolographic analysis (Fig. 3D). These results indicate that mTOR activity is necessary for the synergistic effect of IFN- $\alpha$ and RPM against RCC cells.

Effect of VHL activity on the synergy of IFN- $\alpha$ and RPM. The effect of VHL activity on the synergy of IFN- $\alpha$ and RPM against RCC was also investigated. VHL expression was downregulated in ACHN cells via RNAi and upregulated in A498 cells by transfection with a VHL vector. VHL/HIF expression was confirmed by western blot analysis and RT-PCR (Fig. 4A 
A

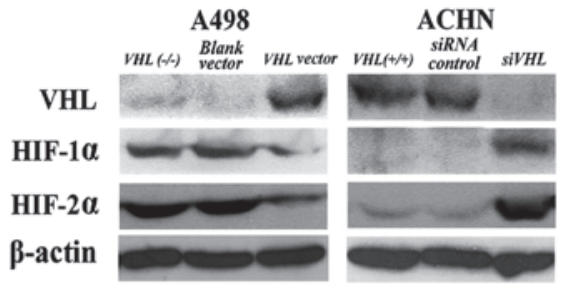

C

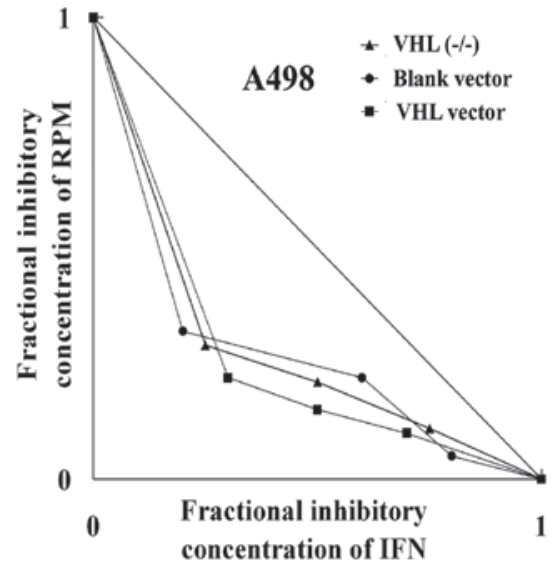

B

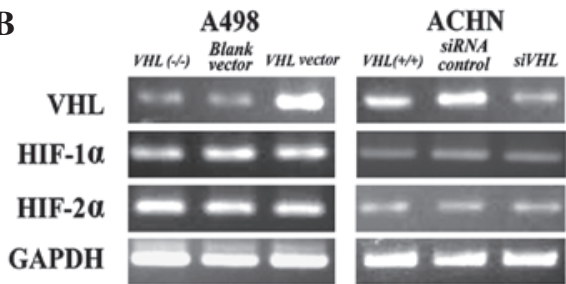

D

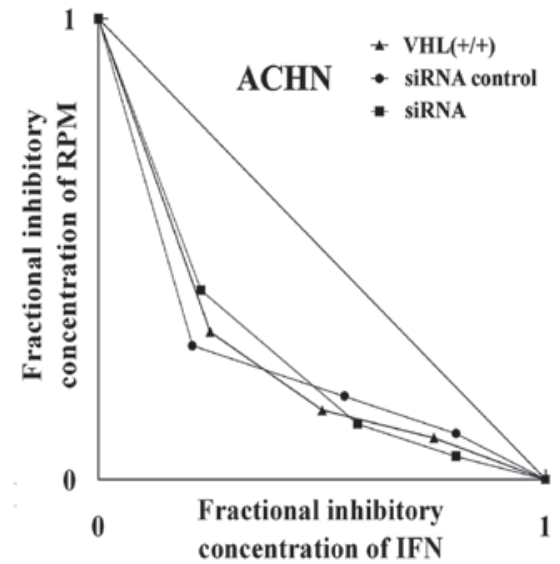

Figure 4. Effect of VHL activity on the synergy of IFN- $\alpha$ and RPM. VHL expression of VHL was downregulated in ACHN cells by RNAi and upregulated in A498 cells by transfection with a VHL vector, as confirmed by (A) western blot analysis and (B) RT-PCR. Isobolographic analysis demonstrated the effect of combination therapy with IFN- $\alpha$ and RPM on (C) A498 and (D) ACHN RCC cell lines. VHL, Von Hippel-Lindau; IFN, interferon; RPM, rapamycin; HIF, hypoxia-inducible transcription factor; RT-PCR, reverse transcription polymerase chain reaction; RCC, renal cell carcinoma.

and B). Since VHL mediates HIF levels via a post-translational mechanism, VHL did not alter the mRNA expression levels of HIF- $1 \alpha$ or $-2 \alpha$. Therefore, the results indicate that regardless of VHL activity, IFN- $\alpha$ enhances the susceptibility of RCC to RPM in all RCC cells tested in the study (Fig. 4C and D). Thus, the synergy of IFN- $\alpha$ and RPM does not depend on VHL activity in RCC cells.

\section{Discussion}

Although a number of clinical trials with various combination chemotherapies have been performed in an attempt to overcome the current limitations of advanced RCC treatment, few have achieved favorable results or prognosis for patients with the disease (25). Therefore, the development of more effective combination chemotherapies for advanced RCC is required.

Promising new combination chemotherapies are usually identified simultaneously with advances in the understanding of oncogenesis. RPM has previously been reported to have immunosuppressant and anticancer effects on a large variety of malignancies, including hepatocellular carcinoma and RCC $(26,27)$. In addition, RPM is well tolerated with minimal side-effects and has shown anticancer activity in patients with androgen-independent prostate cancer (28). Previous studies concerning combination chemotherapy of RPM with chemotherapeutic agents have been performed and combinations of RPM with bevacizumab, sorafenib or 5-fluorouracil have been reported to be promising therapeutic approaches for the treatment of hepatocellular carcinoma (29-33). IFN- $\alpha$ therapy is the most common approach for advanced RCC. However, the synergistic effects of RPM and IFN- $\alpha$ against RCC remain unclear. In the present study, the effect of a combination of IFN- $\alpha$ and RPM on the inhibition of RCC cell growth was analyzed. The results demonstrated that IFN- $\alpha$ and RPM caused dose-dependent inhibition of proliferation and combined treatment with the two agents resulted in synergistic growth suppression in all three RCC cell lines examined. At present, IFN- $\alpha$ is widely administered for the treatment of RCC. The observations of the present study indicate that RPM may be an optimal agent to combine with IFN- $\alpha$ for clinical application against RCC. Since chemotherapy is associated with severe side-effects that usually limit the clinical application, reducing the dosage of IFN- $\alpha$ or RPM is expected to alleviate the associated side-effects but not decrease the synergistic effects of these agents. Therefore, further clinical trials are required to analyze the tolerance towards IFN- $\alpha$ and RPM and to reveal the possible synergy of the two agents in patients with RCC.

The underlying mechanism behind the synergy between IFN- $\alpha$ and RPM in RCC cell lines was further investigated. The molecular mechanism promoting the anticancer effects of RPM is complex, as RPM suppresses the activity of mTOR and the phosphorylation of its downstream effectors, p70S6K and 4E-BP1 (34). The mTOR pathway is considered to be a central regulator in various malignant tumors. There are two distinct functional mTOR complexes. Firstly, mTORC1 consists of mTOR and regulatory-associated protein of mTOR (Raptor) and increases the phosphorylation of p70 S6K/4E-BP1. Secondly, there is mTORC2, which consists of mTOR and rapamycin-insensitive companion of mTOR and increases Akt (also known as protein kinase B) phosphorylation (35). Akt enhances cell growth by alleviating the tuberous sclerosis complex $1 / 2$ suppression of mTOR, allowing the latter to function as part of the mTOR/Raptor complex on p70 S6K and 4E-BP1 (36,37). p70 S6K phosphorylates the S6 protein of the $40 \mathrm{~S}$ ribosomal subunit (38), while translation repressor 
protein 4E-BP1 inhibits translation by binding to the translation initiation factor eIF4E $(39,40)$. Hyperphosphorylation of $4 \mathrm{E}-\mathrm{BP} 1$ disrupts this interaction and results in the activation of translation (41).

In the present study, the role of the mTOR pathway in the synergistic effect of IFN- $\alpha$ and RPM against RCC was investigated. The results indicated that IFN- $\alpha$ and RPM did not affect protein expression in the mTOR pathway. However, each agent individually decreased the phosphorylation of mTOR, p70 S6K, S6 and 4E-BP1 in RCC cells. In addition, IFN- $\alpha$ significantly enhanced the RPM-induced suppression of the mTOR pathway, indicating that the synergy between IFN- $\alpha$ and RPM against RCC depends on the suppression of the mTOR pathway. The effect of mTOR activity on the synergy of IFN- $\alpha$ and RPM was also analyzed. In RCC cells expressing low levels of mTOR, the synergistic growth suppression of the two agents was eliminated and an additive effect was observed. These observations indicate that mTOR activity is important for the synergy of IFN- $\alpha$ and RPM against RCC cells.

Inactivation of the VHL tumor suppressor protein is a common event in clear cell RCC, which is the most common form of kidney cancer. A previous study reported that, in response to IFN- $\alpha$, the exponential growth of wild-type VHL RCC cells was inhibited more than that of VHL-null RCC cells. This observation indicated that VHL inactivation may be involved in IFN- $\alpha$ resistance and that combined immunotherapy with antiangiogenic drugs may be beneficial for patients with a mutated VHL gene $(42,43)$. However, the effect of VHL activity on the synergy of IFN- $\alpha$ and RPM against RCC is unknown. In the present study, A498 was used as the VHL-null RCC cell line, while the other cell lines were wild type for VHL. The results indicated that regardless of VHL activity, synergy of IFN- $\alpha$ and RPM was observed in all RCC cells and, thus, may be independent of VHL activity.

In conclusion, the present study demonstrated that the mTOR pathway plays an important role in the synergistic effect of IFN- $\alpha$ and RPM against RCC cells. The results indicate that blocking the activity of mTOR may provide a novel treatment strategy for patients with RCC. In addition, the suppression of RCC cell growth by IFN- $\alpha$ and RPM may be more effective in RCC cells with high mTOR activity.

\section{References}

1. Pantuck AJ, Zisman A and Belldegrun AS: The changing natural history of renal cell carcinoma. J Urol 166: 1611-1623, 2001.

2. Hartmann JT and Bokemeyer C: Chemotherapy for renal cell carcinoma. Anticancer Res 19: 1541-1543, 1999.

3. Hernberg M, Pyrhönen S and Muhonen T: Regimens with or without interferon-alpha as treatment for metastatic melanoma and renal cell carcinoma: an overview of randomized trials J Immunother 22: 145-154, 1999.

4. Yanai Y, Horie S, Yamamoto K, et al: Characterization of the antitumor activities of IFN-alpha8 on renal cell carcinoma cells in vitro. J Interferon Cytokine Res 21: 1129-1136, 2001.

5. Darnell JE Jr, Kerr IM and Stark GR: Jak-STAT pathways and transcriptional activation in response to IFNs and other extracellular signaling proteins. Science 264: 1415-1421, 1994.

6. Rathmell WK and Godley PA: Recent updates in renal rell carcinoma. Curr Opin Oncol 22: 250-256, 2010.

7. Kremer CL, Klein RR, Mendelson J, et al: Expression of mTOR signaling pathway markers in prostate cancer progression. Prostate 66: 1203-1212, 2006.
8. Hashemolhosseini S, Nagamine Y, Morley SJ, Desrivières S, Mercep L and Ferrari S: Rapamycin inhibition of the G1 to S transition is mediated by effects on cyclin D1 mRNA and protein stability. J Biol Chem 273: 14424-14429, 1998.

9. Nozawa H, Watanabe T and Nagawa H: Phosphorylation of ribosomal p70 S6 kinase and rapamycin sensitivity in human colorectal cancer. Cancer Lett 251: 105-113, 2007.

10. Ruvinsky I, Sharon N, Lerer T, et al: Ribosomal protein S6 phosphorylation is a determinant of cell size and glucose homeostasis. Genes Dev 19: 2199-2211, 2005.

11. Seufferlein T and Rozengurt E: Rapamycin inhibits constitutive p70s6k phosphorylation, cell proliferation, and colony formation in small cell lung cancer cells. Cancer Res 56: 3895-3897, 1996.

12. Grewe M, Gansauge F, Schmid RM, Adler G and Seufferlein T: Regulation of cell growth and cyclin D1 expression by the constitutively active FRAP-p70s6K pathway in human pancreatic cancer cells. Cancer Res 59: 3581-3587, 1999.

13. Cho D, Signoretti S, Regan M, Mier JW and Atkins MB: The role of mammalian target of rapamycin inhibitors in the treatment of advanced renal cancer. Clin Cancer Res 13: 758s-763s, 2007.

14. Luan FL, Ding R, Sharma VK, Chon WJ, Lagman M and Suthanthiran M: Rapamycin is an effective inhibitor of human renal cancer metastasis. Kidney Int 63: 917-926, 2003.

15. Shi Y, Frankel A, Radvanyi LG, Penn LZ, Miller RG and Mills GB: Rapamycin enhances apoptosis and increases sensitivity to cisplatin in vitro. Cancer Res 55: 1982-1988, 1995.

16. Masiello D, Mohi MG, McKnight NC, et al: Combining an mTOR antagonist and receptor tyrosine kinase inhibitors for the treatment of prostate cancer. Cancer Biol Ther 6: 195-201, 2007.

17. Wu WZ, Sun HC, Shen YF, et al: Interferon alpha 2a down-regulates VEGF expression through PI3 kinase and MAP kinase signaling pathways. J Cancer Res Clin Oncol 131: 169-178, 2005.

18. Costa LJ, Gemmill RM and Drabkin HA: Upstream signaling inhibition enhances rapamycin effect on growth of kidney cancer cells. Urology 69: 596-602, 2007.

19. Linehan WM, Lerman MI and Zbar B: Identification of the von Hippel-Lindau (VHL) gene. Its role in renal cancer. JAMA 273: 564-570, 1995.

20. Kaelin WG Jr: Molecular basis of the VHL hereditary cancer syndrome. Nat Rev Cancer 2: 673-682, 2002.

21. Guba M, von Breitenbuch P, Steinbauer M, et al: Rapamycin inhibits primary and metastatic tumor growth by antiangiogenesis: involvement of vascular endothelial growth factor. Nat Med 8: 128-135, 2002.

22. Treins C, Giorgetti-Peraldi S, Murdaca J, MonthouëlKartmann MN and Van Obberghen E: Regulation of hypoxia-inducible factor (HIF)-1 activity and expression of HIF hydroxylases in response to insulin-like growth factor I. Mol Endocrinol 19: 1304-1317, 2005.

23. Liu YT, Shang D, Akatsuka S, et al: Chronic oxidative stress causes amplification and overexpression of ptprz1 protein tyrosine phosphatase to activate beta-catenin pathway. Am J Pathol 171: 1978-1988, 2007.

24. Berenbaum MC: A method for testing for synergy with any number of agents. J Infect Dis 137: 122-130, 1978.

25. Hattori K and Akaza H: New combination chemotherapy in urological cancers. Gan To Kagaku Ryoho 27: 382-387, 2000 (In Japanese).

26. Zhang JF, Liu JJ, Lu MQ, et al: Rapamycin inhibits cell growth by induction of apoptosis on hepatocellular carcinoma cells in vitro. Transpl Immunol 17: 162-168, 2007.

27. Bukowski RM: Metastatic renal cell carcinoma: role of mammalian target of rapamycin inhibitors. Clin Genitourin Cancer 5: 359-361, 2007.

28. Amato RJ, Jac J, Mohammad T and Saxena S: Pilot study of rapamycin in patients with hormone-refractory prostate cancer. Clin Genitourin Cancer 6: 97-102, 2008.

29. Wang Z, Zhou J, Fan J, et al: Effect of rapamycin alone and in combination with sorafenib in an orthotopic model of human hepatocellular carcinoma. Clin Cancer Res 14: 5124-5130, 2008.

30. Huynh H, Chow PK, Palanisamy N, et al: Bevacizumab and rapamycin induce growth suppression in mouse models of hepatocellular carcinoma. J Hepatol 49: 52-60, 2008.

31. Shi Y and August DA: A new trick for an old drug: mTOR inhibitor rapamycin augments the effect of fluorouracil on hepatocellular carcinoma by inducing cell senescence. Cancer Biol Ther 7: 397-398, 2008. 
32. Pectasides D, Pectasides E, Papaxoinis G, et al: Combination chemotherapy with docetaxel, vinorelbine and estramustine phosphate in metastatic androgen-resistant prostate cancer: a single institution experience. Anticancer Res 29: 769-775, 2009.

33. Vaishampayan UN, Marur S, Heilbrun LK, et al: Phase II trial of capecitabine and weekly docetaxel for metastatic castrate resistant prostate cancer. J Urol 182: 317-323, 2009.

34. Hou G, Xue L, Lu Z, Fan T, Tian F and Xue Y: An activated $\mathrm{mTOR} / \mathrm{p} 70 \mathrm{~S} 6 \mathrm{~K}$ signaling pathway in esophageal squamous cell carcinoma cell lines and inhibition of the pathway by rapamycin and siRNA against mTOR. Cancer Lett 253: 236-248, 2007.

35. Fan QW and Weiss WA: Inhibition of PI3K-Akt-mTOR signaling in glioblastoma by mTORC1/2 inhibitors. Methods Mol Biol 821 349-359, 2012.

36. Fingar DC, Salama S, Tsou C, Harlow E and Blenis J: Mammalian cell size is controlled by mTOR and its downstream targets S6K1 and 4EBP1/eIF4E. Genes Dev 16: 1472-1487, 2002.

37. Rosner M and Hengstschläger M: Nucleocytoplasmic localization of p70 S6K1, but not of its isoforms p 85 and $\mathrm{p} 31$, is regulated by TSC2/mTOR. Oncogene 30: 4509-4522, 2011.
38. Terzis G, Spengos K, Mascher H, Georgiadis G, Manta P and Blomstrand E: The degree of p70 S6k and S6 phosphorylation in human skeletal muscle in response to resistance exercise depends on the training volume. Eur J Appl Physiol 110: 835-843, 2010.

39. Pullen $\mathrm{N}$ and Thomas G: The modular phosphorylation and activation of p70s6k. FEBS Lett 410: 78-82, 1997.

40. Hinnebusch AG: Translational homeostasis via eIF4E and 4E-BP1. Mol Cell 46: 717-719, 2012.

41. Tait S, Dutta K, Cowburn D, Warwicker J, Doig AJ and McCarthy JE: Local control of a disorder-order transition in 4E-BP1 underpins regulation of translation via eIF4E. Proc Natl Acad Sci USA 107: 17627-17632, 2010.

42. Pause A, Belsham GJ, Gingras AC, et al: Insulin-dependent stimulation of protein synthesis by phosphorylation of a regulator of 5'-cap function. Nature 371: 762-767, 1994.

43. Perier A, Fregni G, Wittnebel S, et al: Mutations of the von Hippel-Lindau gene confer increased susceptibility to natural killer cells of clear-cell renal cell carcinoma. Oncogene 30: 2622-2632, 2011. 